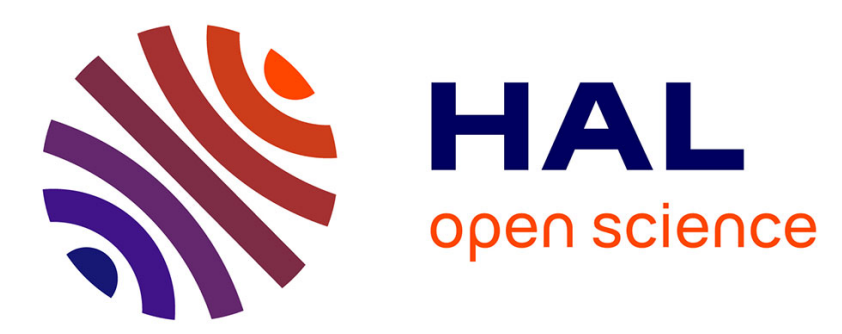

\title{
A CVD Diamond Beam Telescope for Charged Particle Tracking
}

W. Adam, E. Berdermann, P. Bergonzo, W. de Boer, F. Bogani, E. Borchi, A. Brambilla, M. Bruzzi, C. Colledani, J. Conway, et al.

\section{> To cite this version:}

W. Adam, E. Berdermann, P. Bergonzo, W. de Boer, F. Bogani, et al.. A CVD Diamond Beam Telescope for Charged Particle Tracking. 2001 IEEE Nuclear Science Symposium (NSS) and Medical Imaging Conference (MIC), Nov 2001, San Diego, United States. N17.1-5 p. in2p3-00013641

\section{HAL Id: in2p3-00013641 https://hal.in2p3.fr/in2p3-00013641}

Submitted on 30 Jul 2002

HAL is a multi-disciplinary open access archive for the deposit and dissemination of scientific research documents, whether they are published or not. The documents may come from teaching and research institutions in France or abroad, or from public or private research centers.
L'archive ouverte pluridisciplinaire HAL, est destinée au dépôt et à la diffusion de documents scientifiques de niveau recherche, publiés ou non, émanant des établissements d'enseignement et de recherche français ou étrangers, des laboratoires publics ou privés. 


\section{A CVD Diamond Beam Telescope for Charged Particle Tracking}

THE RD42 COLLABORATION

W. Adam ${ }^{1}$, E. Berdermann ${ }^{2}$, P. Bergonzo ${ }^{3}$, W. de Boer ${ }^{4}$, F. Bogani ${ }^{5}$, E. Borchi ${ }^{6}$, A. Brambilla ${ }^{3}$, M. Bruzzi ${ }^{6}$, C. Colledani ${ }^{7}$, J. Conway ${ }^{8}$, P. D' Angelo ${ }^{9}$, W. Dabrowski ${ }^{10}$, P. Delpierre ${ }^{11}$, W. Dulinski ${ }^{7}$, J. Doroshenko ${ }^{8}$, M. Doucet ${ }^{12}$, B. van Eijk ${ }^{13}$, A. Fallou ${ }^{11}$, P. Fischer ${ }^{14}$, F. Fizzotti ${ }^{15}$, D. Kania ${ }^{16}$, K.K. Gan ${ }^{16}$, E. Grigoriev ${ }^{4}$, G. Hallewell ${ }^{11}$, S. Han ${ }^{16}$, F. Hartjes ${ }^{13}$, J. Hrubec ${ }^{1}$, D. Husson ${ }^{7}$, H. Kagan ${ }^{16}$, J. Kaplon ${ }^{17}$, R. Kass ${ }^{16}$, M. Keil ${ }^{14}$, K.T. Knöpfle ${ }^{18}$, T. Koeth ${ }^{8}$, M. Krammer ${ }^{1}$, S. Meuser ${ }^{14}$, A. Logiudice ${ }^{15}$, L. mac Lynne ${ }^{8}$, C. Manfredotti ${ }^{15}$, D. Meier ${ }^{17}$, * , D. Menichelli ${ }^{6}$, M. Mishina ${ }^{19}$, L. Moroni ${ }^{9}$, J. Noomen $^{13}$, A. $\mathrm{Oh}^{17}$, L.S. Pan $^{16}$, M. Pernicka ${ }^{1}$, L. Perera ${ }^{8}$, J.L. Riester ${ }^{7}$, S. Roe ${ }^{17}$, A. Rudge ${ }^{17}$, J. Russ ${ }^{20}$, S. Sala ${ }^{9}$, M. Sampietro ${ }^{21}$, S. Schnetzer ${ }^{8}$, S. Sciortino ${ }^{6}$, H. Stelzer ${ }^{2}$, R. Stone ${ }^{8}$, B. Suter ${ }^{20}$, W. Trischuk ${ }^{22}$, D. Tromson ${ }^{3}$, E. Vittone ${ }^{15}$, P. Weilhammer ${ }^{17}$, N. Wermes ${ }^{14}$, M. Wetstein $^{8}$, W. Zeuner ${ }^{12}$, M. Zoeller $^{16}$

${ }^{1}$ HEPHY, Vienna, Austria- ${ }^{2}$ GSI, Darmstadt, Germany- ${ }^{3}$ LETI/DEIN/SPE/CEA Saclay, France- ${ }^{4}$ Universität Karlsruhe, Germany- ${ }^{5}$ LENS, Florence, Italy- ${ }^{6}$ University of Florence, Italy- ${ }^{7}$ LEPSI, IN2P3/CNRS-ULP, Strasbourg, France8 Rutgers University, Piscataway, U.S.A.- ${ }^{9}$ INFN, Milano, Italy- ${ }^{10}$ UMM, Cracow, Poland- ${ }^{11}$ CPPM, Marseille, France12 II.Inst. Exp. Phy., Hamburg, Germany- ${ }^{13}$ NIKHEF, Amsterdam, Netherlands- ${ }^{14}$ Universität Bonn, Germany${ }^{15}$ University of Torino, Italy- ${ }^{16}$ Ohio State University, Columbus, U.S.A.- ${ }^{17}$ CERN, Geneva, Switzerland- ${ }^{18}$ MPI Kernphysik, Heidelberg, Germany- ${ }^{19}$ FNAL, Batavia, U.S.A.- ${ }^{20}$ Carnegie-Mellon University, Pittsburgh, U.S.A.${ }^{21}$ Polytechnico Milano, Italy- ${ }^{22}$ University of Toronto, Canada

Abstract-CVD diamond is a radiation hard sensor material which may be used for charged particle tracking near the interaction region in experiments at high luminosity colliders. The goal of the work described here is to investigate the use of several detector planes made of CVD diamond strip sensors for charged particle tracking. Towards this end a tracking telescope composed entirely of CVD diamond planes has been constructed. The telescope was tested in muon beams and its tracking capability has been investigated.

Keywords-Diamond strip detector, beam telescope, diamond chemical vapour deposition (CVD), charged particle tracking, radiation hardness

\section{INTRODUCTION}

$\mathrm{I}_{\mathrm{i}}^{\mathrm{N}}$ $\mathrm{N}$ order to measure vertices from particles decaying near the interaction region, tracking in experiments at the Large Hadron Collider (LHC) requires fast and radiation hard position sensitive detectors. Close to the interaction region devices must operate at high particle flux and fluence. Detectors based on CVD diamond are an option for the innermost layer of trackers since diamond is a fast and radiation hard sensor material.

CVD diamond strip- and pixel detectors have been extensively tested in the past [1], [2], [3]. The recent progress in industrial production of CVD diamond allowed us to assemble several layers of diamond strip sensors into a diamond beam telescope. This is the first time particle tracking has been performed using hits measured in diamonds only. The work described here was carried out by RD42 a research and development project based at CERN. The goal of the RD42 collaboration is to investigate and develop diamond detectors for particle tracking at the LHC [4].

\section{CVD DiAmond STRIP DETECTORS}

The diamond material used here was produced by chemical vapor deposition (CVD) at De Beers Industrial Diamonds [5]. The material was processed to a thickness of $\approx 500 \mu \mathrm{m}$ and cut from 6 " diamond disks to a final size of $2 \times 2 \mathrm{~cm}^{2}$. The diamonds were metallized with a solid electrode on one side and 256 narrow strips and four $2 \times 8 \mathrm{~mm}^{2}$ pads (for tests in the lab) on the other side. The strips had a width of $25 \mu \mathrm{m}$ at a strip and readout pitch of $50 \mu \mathrm{m}$. The detectors were wire bonded (dc-coupled) to VA2 readout electronics [6]. One VA2 contains 128 charge sensitive pre-amplifiers and shapers with sample-and-hold and a multiplexer for sequential readout. Fig. 1 shows a photograph

\footnotetext{
*corresponding author: Dirk.Meier@cern.ch
}

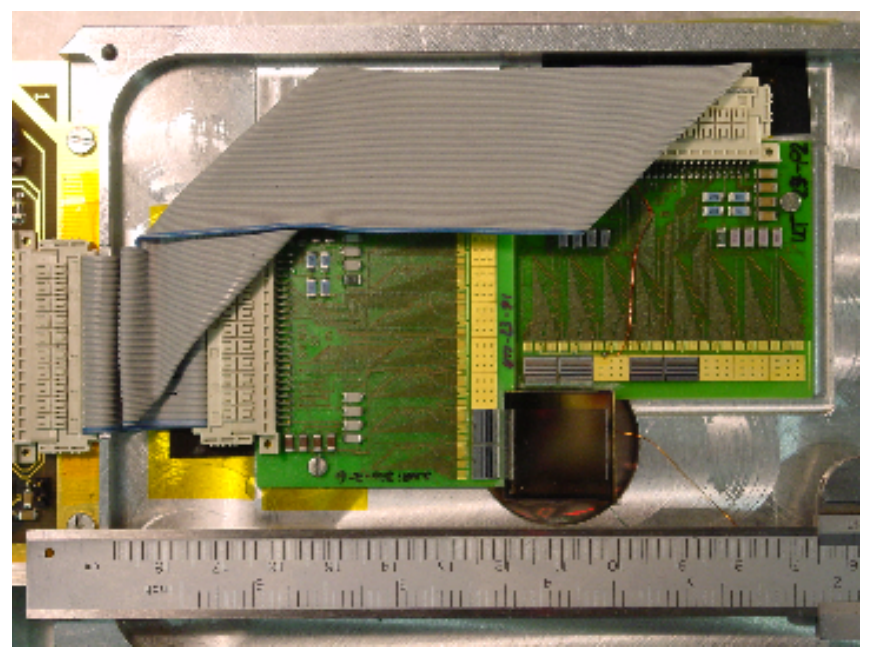

Fig. 1. Photograph of two hybrids carrying diamond strip detectors and readout chips. The diamond detector can be seen centered over the circular hole in the aluminium frame.

of two $2 \times 2 \mathrm{~cm}^{2}$ diamond detectors on hybrids inside an aluminium frame.

\section{RESUlTS FROM ${ }^{90} \mathrm{SR} \beta$-SOURCE TESTS}

Fig. 2 shows the distribution of charge signals measured on the solid test electrode in one of the diamond planes using electrons from a ${ }^{90} \mathrm{Sr} \beta$-source. The signal in the diamond detector is Landau-like distributed with a clear separation from zero, a most probable signal of $6500 e$ and a mean signal of $7911 e$ which corresponds to a charge collection distance of $220 \mu \mathrm{m}$. The scale was calibrated by measuring the photoabsorption peak at $59.5 \mathrm{keV}$ from ${ }^{241} \mathrm{Am}$ in a silicon diode.

Fig. 3 shows the mean collected signal charge as a function of the applied voltage in the range from $-500 \mathrm{~V}$ to $+500 \mathrm{~V}$ (corresponding to an electric field of $-1 \mathrm{~V} / \mu \mathrm{m}$ to $+1 \mathrm{~V} / \mu \mathrm{m}$ ). The mean signal is symmetric around zero and follows an " $\mathrm{S}$ curve". The mean signal saturates near $\pm 1 \mathrm{~V} / \mu \mathrm{m}$ due to velocity saturation of the charge carriers. We chose to operate all diamonds described here at $+1 \mathrm{~V} / \mu \mathrm{m}$. The figure also shows the leakage current as a function of the voltage. The leakage current also follows a symmetric "S-curve" with $\pm 35 \mathrm{pA} / \mathrm{mm}^{2}$ at $\pm 1 \mathrm{~V} / \mu \mathrm{m}$ - a very low current density. For the VA2 readout used here we estimate a noise of $30 e \mathrm{ENC}$ per channel due to leakage 


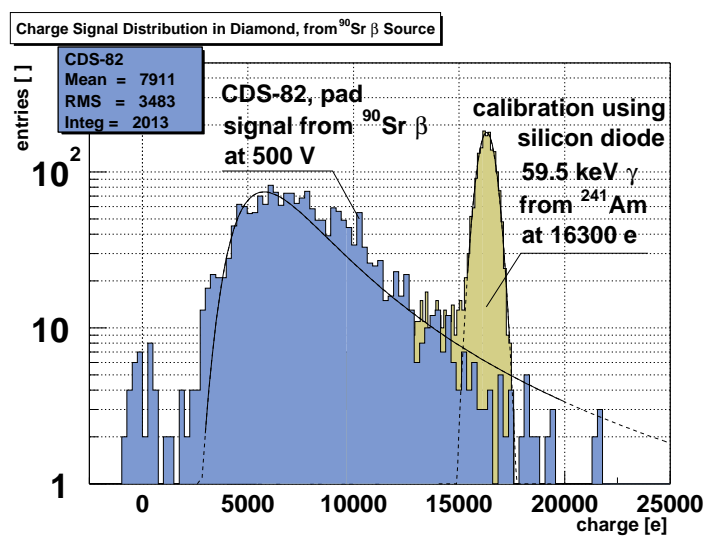

Fig. 2. Foreground: distribution of the charge measured on a solid electrode in one of the diamond planes using electrons from a ${ }^{90} \mathrm{Sr} \beta$-source. Background: distribution of the charge signal from a silicon diode using $59.5 \mathrm{keV}$ photons from ${ }^{241} \mathrm{Am}$. The same electronic amplification channel was used.

current of $35 \mathrm{pA}$ in $20 \mathrm{~mm}$ long strips.

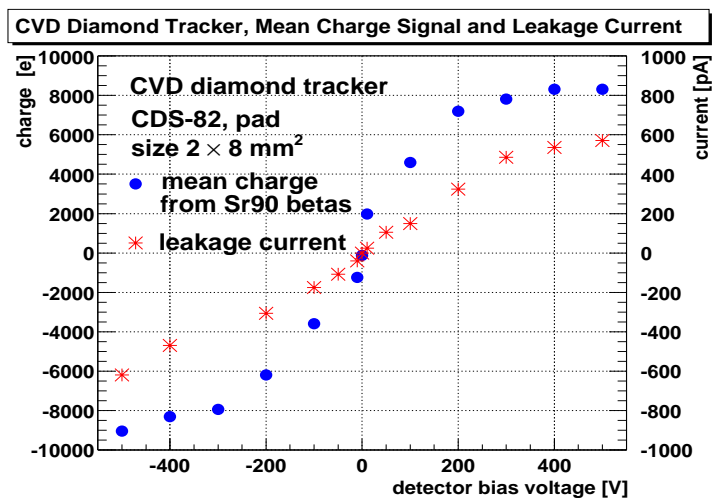

Fig. 3. Mean charge collected and leakage current, measured as a function of the detector bias voltage (at room temperature). The mean charge was measured using a ${ }^{90} \mathrm{Sr} \beta$-source.

Table I lists the CVD diamond detectors used here together with their dimensions, type of contacts, mean charge collected measured with a ${ }^{90} \mathrm{Sr} \beta$-source, $\bar{Q}_{\mathrm{col}}^{90} \mathrm{Sr}$, at $\approx 1 \mathrm{~V} / \mu \mathrm{m}$ and corresponding charge collection distance, $d=\bar{Q}_{\text {col }}^{90} \mathrm{Sr}[e] / 36 e / \mu \mathrm{m}$. It can be seen that $d$ ranges from $164 \mu \mathrm{m}$ to $234 \mu \mathrm{m}$ - the later is a typical value for todays sensors from production reactors. We note that the values are given after "pumping", the stable and reproducible state of the diamond detector after a minimum of 10 Gy absorbed dose from ${ }^{90} \mathrm{Sr}$ electrons [7].

\section{Telescope Experimental Setup In the BeAm}

The seven $2 \times 2 \mathrm{~cm}^{2}$ diamonds used in the telescope were mounted on hybrids [Fig. 1] and fixed above each other in two aluminium frames (4 hybrids in frame 1, 3 hybrids in frame 2). Every second plane was rotated by $90^{\circ}$ which allowed one to measure two orthogonal coordinates of the particle track. Fig. 4 shows a schematic side view of the complete setup in the beam: The two frames were fixed $120 \mathrm{~mm}$ apart (150 mm pitch) and aligned such that beam particles traversed all planes perpendicularly. The seven $2 \times 2 \mathrm{~cm}^{2}$ diamond detectors formed the beam telescope ("reference planes"). The two $1 \mathrm{~cm}^{2} \mathrm{di}$ amond detectors were mounted inside this telescope ("planes
TABLE I

List of CVD diamond strip detectors tested, with thickness, size, type of contacts, the mean collected charge measured with ${ }^{90} \mathrm{Sr}$, and the corresponding charge collection distance. The first seven diamonds made up the telescope. ${ }^{*}$ ) The results for CD83 are estimated from the unpumped values.

\begin{tabular}{|c|c|c|c|c|c|}
\hline name & $\begin{array}{c}\text { thick } \\
{[\mu \mathrm{m}]}\end{array}$ & $\begin{array}{c}\text { size } \\
{[\mathrm{cm} \times \mathrm{cm}]}\end{array}$ & $\begin{array}{c}\text { con- } \\
\text { tacts }\end{array}$ & $\begin{array}{c}\bar{Q}_{\text {col }}^{90}[\mathrm{Sr} \\
{[e]}\end{array}$ & $\begin{array}{c}d \\
{[\mu \mathrm{m}]}\end{array}$ \\
\cline { 1 - 4 } CD83-P1 & 485 & $2 \times 2$ & $\mathrm{Al}$ & $7605^{*}$ & $211^{*}$ \\
\hline CD83-P2 & 490 & $2 \times 2$ & $\mathrm{Al}$ & & \\
\hline UT24-P1 & 540 & $2 \times 2$ & $\mathrm{CrAu}$ & 5915 & 164 \\
\hline UT24-P2 & 540 & $2 \times 2$ & $\mathrm{CrAu}$ & 6510 & 181 \\
\hline UT23-P1 & 540 & $2 \times 2$ & $\mathrm{CrAu}$ & 7096 & 197 \\
\hline UT23-P2 & 540 & $2 \times 2$ & $\mathrm{CrAu}$ & 7068 & 196 \\
\hline CD82-P2 & 510 & $2 \times 2$ & $\mathrm{CrAu}$ & 8200 & 228 \\
\hline \hline CD88 & 470 & $1 \times 1$ & $\mathrm{Al}$ & 8370 & 233 \\
\hline CD90 & 470 & $1 \times 1$ & $\mathrm{Al}$ & 8416 & 234 \\
\hline
\end{tabular}

under-test"). Several planes of silicon detectors - measured in previous tests [8], [9] - were mounted along the beam in order to project tracks into them.

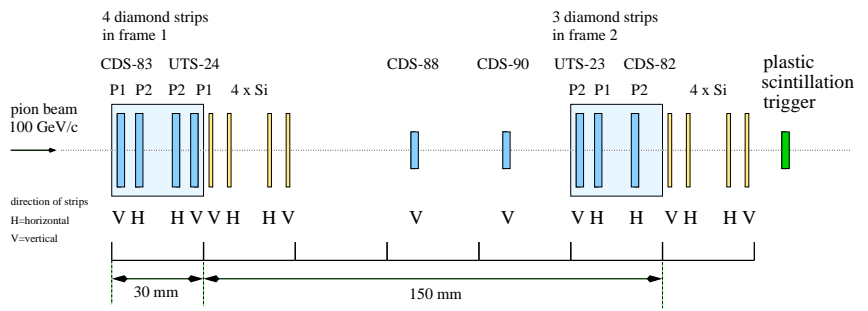

Fig. 4. Schematic side view of diamond- and silicon strip trackers in the test beam. The orientation of the strips is horizontal $(\mathrm{H})$ and vertical $(\mathrm{V})$.

A $7 \times 7 \mathrm{~mm}^{2}$ large plastic scintillator with photo-multiplier readout, mounted downstream, behind the second frame was used to trigger the readout of all detector planes. The scintillator was smaller than the diamond reference planes and most beam triggers were related to particles traversing the detector planes.

Fig. 5 shows, the pedestal, noise and signal from diamond detector CD90. The analysis algorithm performed pedestal subtraction, common mode correction and measured the remaining noise on channels where the later is shown in equivalent noise charge, ENC, in units of the ADC and in electrons. The calibration between ADC and electrons was measured using a silicon diode and photons from ${ }^{241} \mathrm{Am}$. The figure shows that the noise on all strips is fairly uniform ranging between $80 e$ and $100 e$. The noise measurement is important in order to discriminate signals which are related to charged particles. For each channel a discrimination threshold of 5 times the $r m s$ noise was chosen. In silicon detectors the charge is induced on one or two strips depending on the position of the track between the strips. Fig. 5 (bottom) shows the distribution of 2-strip signal charge on 128 strips where the relative number of entries is given by the box-size in each bin of the two-dimensional histogram. The mean signal values for each strip are overlayed - the signals ob- 
tained on strips are uniformly distributed over all strips and have a mean value of $8640 \mathrm{e}$. The projection of the two dimensional histogram onto its ordinate is shown in Fig. 6 together with the distribution of signal charge from single strips. The signals are Landau-like distributed with a most probable value of $6200 e$, and a mean value of $8640 e$ for 2 -strip signals. There are a few entries near zero due to noise hits. The mean value is $8770 e$ if one excludes these entries in calculating the mean signal. The hit position in the detector perpendicular to the strips can be measured using various methods. For this analysis we always used the 2-strip center-of-gravity method for calculating the hit position.
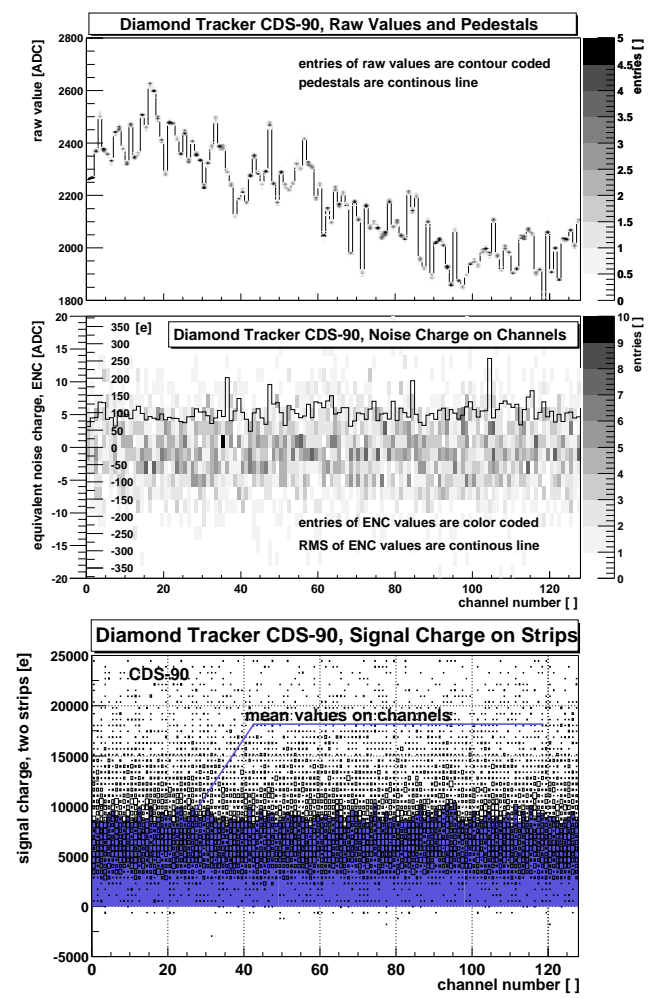

Fig. 5. Top: Raw ADC values from 128 strips of diamond detector CD90. Middle: Noise on strips. Bottom: Signal distribution on strips and their mean signals (overlayed).

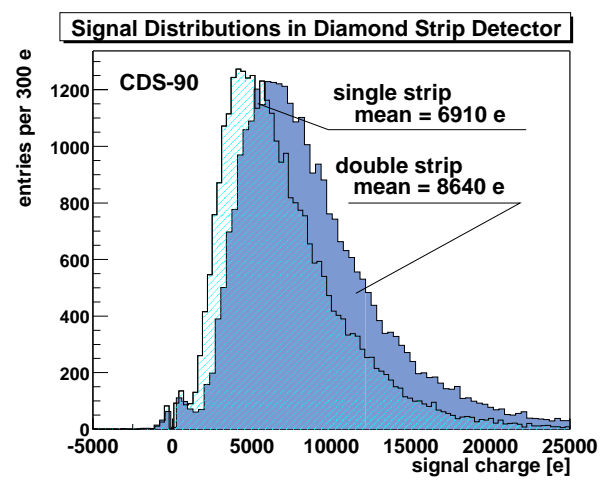

Fig. 6. Signal distributions measured in the beam, for single strips (light) and two strips (dark).

\section{Beam Test Results}

\section{A. Alignment of Planes}

For each beam event a minimum of 4 hits $(2$ hits in horizontal strips and 2 hits in vertical strips) was required to perform a track fit. In each plane only the hit containing the highest signal charge was used. A maximum of 7 hits (one hit per plane allowed) were used. A straight line fit was performed and projected into planes under test in order to obtain residuals, the difference between the projection and the position of the hit measured by the plane.

The reference planes were aligned with respect to each other such that the distribution of residuals along the direction of the strips had zero mean and zero angle. In order to test the alignment each of the diamond planes was checked with respect to four silicon planes. Fig. 7 shows the distribution of residuals obtained from one of the diamond planes with respect to four silicon planes. The distribution along the strips ( $v$-coordinate) shows zero offset and zero tilt confirming correct alignment. The distribution of residuals perpendicular to the strips ( $u$-coordinate) is important since outlying residuals reveal regions where strips or bond-wires may be disconnected (floating) or shorted to adjacent strips or bond-wires. The figure shows two regions of outlying residuals (one at $u \approx 0 \mu \mathrm{m}$, and one at $u \approx 500 \mu \mathrm{m})$.
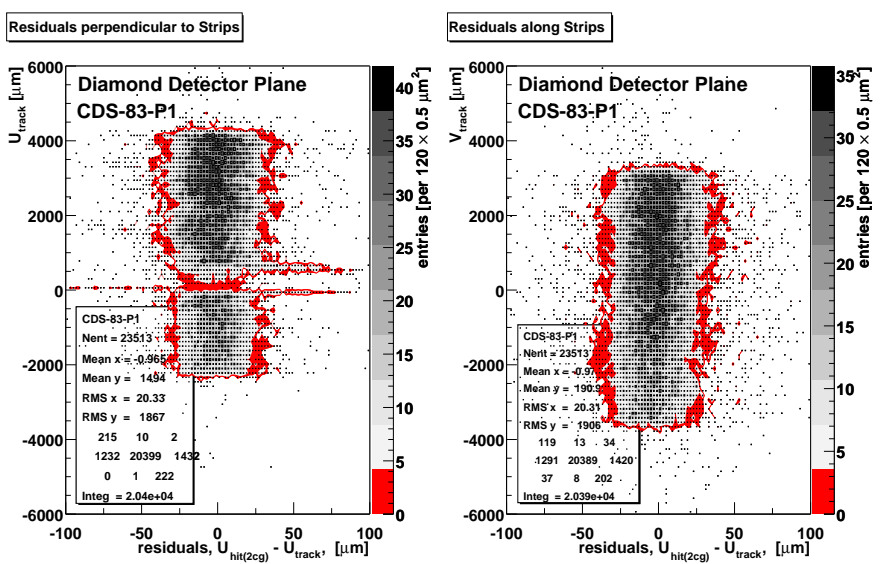

Fig. 7. Residual distributions in diamond strip detector CD83-P1, perpendicular to the strips (left) and along the strips (right).

\section{B. Residuals}

The projection of the data [Fig. 7] onto the abscissa is the residual distribution. The residuals are normal distributed around zero and a Gaussian fit to the distribution gives the spatial resolution, $\sigma$, (convoluted by tracking precision). Using four planes of silicon we measured residual distributions for all diamonds and list $\sigma$ in Table II. The $\sigma$ range from $14.2 \mu \mathrm{m}$ to $16.4 \mu \mathrm{m}$. The silicon tracks had a precision of below $2 \mu \mathrm{m}$ which is small compared to $\sigma$ measured in the diamonds.

\section{Hit Finding Efficiency}

The goal here is to quantify the ability of tracking with diamond detectors over the entire sensor area. This characterizes 
the quality of the charge collection properties of the CVD diamond as well as the quality of the strips, wire-bonds, and readout electronics. We define the hit finding efficiency of a plane by the number of hits measured within $\pm 100 \mu \mathrm{m}$ of the track divided by the number of tracks projected into the plane. The hit finding efficiency, $\varepsilon_{\text {noCuts }}$, was measured for each plane as the number of entries inside the displayed region [Fig. 7] divided by the total number of entries including over- and underflows. For CD83-P1 [Fig. 7] one obtains a hit finding efficiency of $20399 / 23513=86.7 \%$. A "reasonable" geometrical cut can be applied in order to select a fiducial detector region and measure its efficiency, $\varepsilon_{\text {fiducial }}$. Table II lists results from the diamond strip detectors and one silicon strip detector. The hit finding efficiencies according to our definition were measured without geometrical cuts, $\varepsilon_{\text {noCuts }}$, and in a fiducial region, $\varepsilon_{\text {fiducial }}$, by excluding regions which had 'obvious' defects (recognized by their out-lying or low-lying residuals). From the figure one sees that about $3 \%$ of the silicon tracks project outside the diamond. The diamond efficiencies in the table are therefore underestimated by about $3 \%$. We find efficiencies ranging from $73 \%$ to $99 \%$ in the diamond planes and $\approx 99 \%$ in the silicon planes. In order to quantify the quality for a track we calculate for each track fit $\chi^{2}=\sum_{\text {hit, } j}$ residual $_{j}^{2} /$ resolution $_{j}^{2}$. Fig. 8 shows the distributions of $\chi^{2}$ obtained from track fits, for hits in $4,5,6$, and 7 diamond planes corresponding to $0,1,2$, and 3 numbers of degree of freedom, NDF. The entries of the histograms shown, indicate how abundant tracks with various NDF occured. The $\chi^{2}$ was used to select "good" tracks: If $\chi^{2}$ is chosen too low then good tracks can be excluded, if $\chi^{2}$ is chosen to large then 'bad' tracks containing outlying hits can be included. Effects from multiple scattering or effects from several charged tracks are considered to be negligible here.

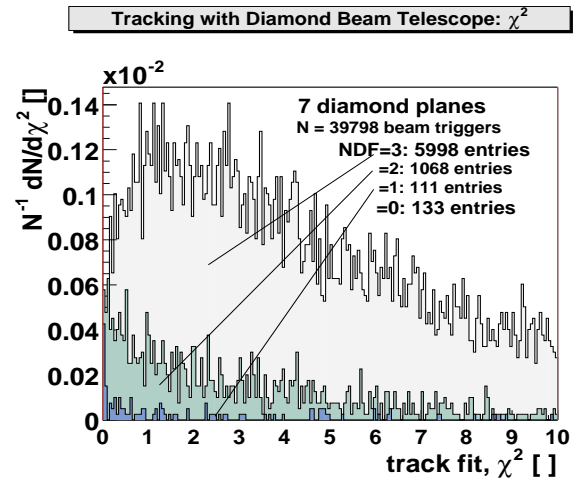

Fig. 8. Distributions of $\chi^{2}$ obtained from track fits, for hits in $4,5,6$, and 7 diamond planes corresponding to $0,1,2$, and 3 numbers of degree of freedom, NDF

\section{Projection into a Silicon Plane}

The goal here is to study a silicon strip detector using the tracks reconstructed by the diamond beam telescope. The behaviour of the silicon detectors as part of the silicon beam telescope [8], [9] is understood: We assume the most probable signal $\hat{Q}_{300 \mu \mathrm{m} \mathrm{Si}}^{\mathrm{mip}}=22500 e$ for two strips in $300 \mu \mathrm{m}$ thick silicon sensors for minimum ionizing charged particles. We measure the mean signal 1.18 times larger than $\hat{Q}_{300 \mu \mathrm{m} \mathrm{Si}}^{\mathrm{mip}}$ and
TABLE II

Results from diamond trackers in the beam, 2-strip mean signal-to-noise, $r m s, \sigma$, of residuals, and hit finding efficiency, $\varepsilon_{\text {noCuts }}$, without geometrical cuts and with geometrical cuts ( $\varepsilon_{\text {fiducial }}$, in fiducial regions).

\begin{tabular}{|c|c|c|c|c|c|}
\hline name & $\begin{array}{c}\text { thick } \\
{[\mu \mathrm{m}]}\end{array}$ & $\bar{S}_{2}: N$ & $\begin{array}{c}\sigma \\
{[\mu \mathrm{m}]}\end{array}$ & $\begin{array}{c}\varepsilon_{\text {noCuts }} \\
{[\%]}\end{array}$ & $\begin{array}{c}\varepsilon_{\text {fiducial }} \\
{[\%]}\end{array}$ \\
\hline CD83-P1 & 485 & $>30$ & 16.4 & 86.7 & 93.3 \\
\hline CD83-P2 & 490 & $>20$ & 16.2 & 81.3 & 87.9 \\
\hline UT24-P1 & 540 & 43 & 15.0 & 87.3 & 87.6 \\
\hline UT24-P2 & 540 & $>10$ & 16.2 & $>70.1$ & $>70.1$ \\
\hline UT23-P1 & 540 & $>15$ & 14.4 & 78.4 & 80.8 \\
\hline UT23-P2 & 540 & $>10$ & 14.8 & 73.6 & 77.2 \\
\hline CD82-P2 & 510 & 45 & 14.2 & 76.3 & 88.8 \\
\hline \hline CD88 & 470 & 88 & 15.4 & 95.9 & 96.3 \\
\hline CD90 & 470 & 92 & 15.9 & 95.6 & 97.5 \\
\hline silicon & 300 & 194 & 2.2 & 98.6 & 98.6 \\
\hline
\end{tabular}

therefore derive $\bar{Q}_{300 \mu \mathrm{m} \mathrm{Si}}^{\mathrm{mip}}=26650$ e. In silicon the signal is separated from zero and the signal distribution begins at about $0.7 \times \hat{Q}_{300 \mu \mathrm{m} \mathrm{Si}}^{\mathrm{mip}}=15750 e$. We measure a most probable signal to noise of $\hat{Q}_{300 \mu \mathrm{m} \mathrm{Si}}^{\mathrm{mip}}: \mathrm{ENC}=160: 1$ and derive a noise of $140 e \mathrm{ENC}$ on a single strip. We previously measured the (intrinsic) spatial resolution of the silicon detectors of $1.4 \mu \mathrm{m}$ using the non-linear $\eta$-method [9]. Fig. 9 shows the "transparent" 2-strip signal from a silicon sensor. The signal was measured on the two strips nearest to the track, where the track was determined by the diamond beam telescope. The data is shown in four representations: a two dimensional plot where the mean signal charge is grey scale coded over the area of the silicon sensor: in this plot one can see the area into which tracks are projected, the outer shape is determined by the scintillation beam trigger of $7 \times 7 \mathrm{~mm}^{2}$. Inside this area some regions have no or few entries due to missing strips or broken electronics in the diamond planes. The same data is shown as a two dimensional histogram projected onto the $u$ - and onto the $v$-coordinate. The lower right histogram shows the 2-strip transparent signal distribution with a most probable signal at $22500 e$. The distribution is slightly smeared out at the rising edge of the signal distribution. We observe $5 \%$ of the entries between 0 and $10000 e$ indicating that $95 \%$ of the tracks from the diamond telescope give a hit in the silicon.

\section{E. Tracking Precision}

The tracking precision of the diamond telescope was derived using the spatial resolution measured in the diamond planes [Table II]. Fig. 9 (left) shows the precision as a function of the position along the beam in horizontal direction using vertical strips and in vertical direction using horizontal strips. The highest precision is inside the telescope, about half way, centered between the planes. For the 4 horizontal strip planes the precision is symmetric reaching $7 \mu \mathrm{m}$ at the center, for the 3 vertical strip planes the precision is slightly asymmetric. Outside of the telescope the precision decreases. Fig. 10 (right) shows two residual distributions measured in two silicon strip sensors with horizontal $(\mathrm{H})$ and vertical $(\mathrm{V})$ strips. The distributions are centered 
A Silicon Strip Detector, 'Seen' by Diamond Beam Telescope
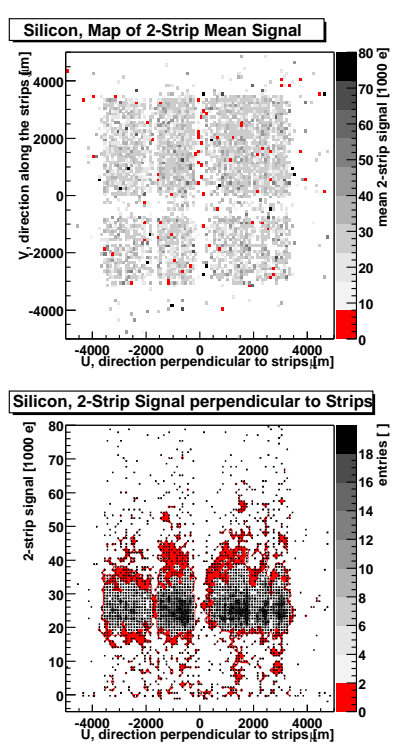
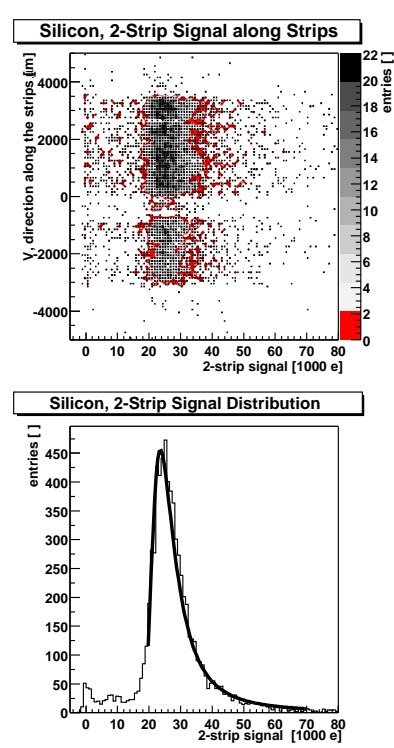

Fig. 9. Signal distribution in a silicon strip detector plane. The signals were measured on the two strips nearest to the track ("transparent" analysis). The tracks were reconstructed by the diamond beam telescope.

around zero with an $\mathrm{rms}$ of $18.2 \mu \mathrm{m}$ for H-strips and $20.7 \mu \mathrm{m}$ for V-strips. The residuals are dominated by the tracking precision. These two planes were mounted about $3 \mathrm{~cm}$ outside of the diamond beam telescope as shown in Fig. 10. The rms for $\mathrm{H}$-silicon strips is smaller than for $\mathrm{V}$-silicon strips due to two $\mathrm{H}$ reference planes but only one $\mathrm{V}$-reference plane near the silicon planes.
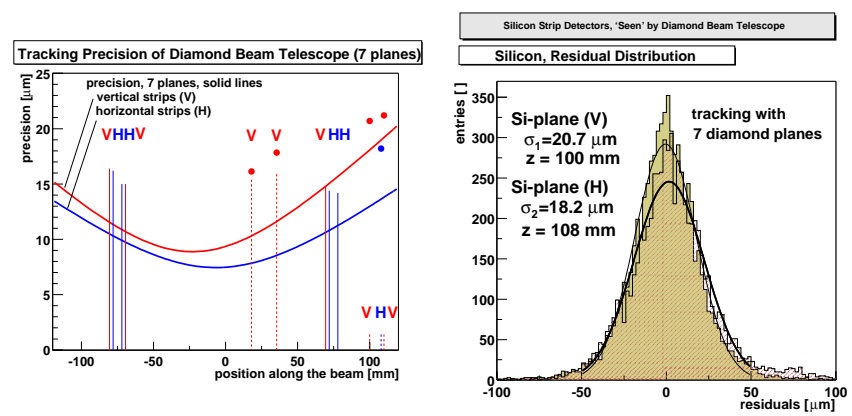

Fig. 10. Left: Tracking precision as a function of the position along the beam. The positions of the planes are given by vertical lines, the length gives the measured spatial resolution. The points show precision measurements using planes under test. Right: Residual distributions in two silicon strip detector planes with horizontal $(\mathrm{H})$ and vertical $(\mathrm{V})$ strips. The tracks were reconstructed by the diamond beam telescope.

\section{SUMMARY}

Seven CVD diamond strip detectors have been assembled into a beam telescope. Two additional diamonds strip trackers and several silicon strip detectors were used for tests. The telescope was tested in muon beams and its tracking capability has been investigated. The mean signals from the diamonds were in the range from $5915 e$ to $8416 e$, corresponding to charge col-

lection distances of $164 \mu \mathrm{m}$ and $234 \mu \mathrm{m}$ measured with ${ }^{90} \mathrm{Sr}$. In the beam we measured mean signal-to-noise ranging from 10-to- 1 to 92 -to- 1 using VA2 readout electronics. The spatial resolution on these diamond strip detectors is near digital resolution using a 2-strip center-of-gravity position finding method. Hit finding efficiencies from $73 \%$ up to $99 \%$ were measured for $5 \times$ noise thresholds and hits on tracks inside $\pm 100 \mu \mathrm{m}$. Using the seven planes we reconstructed straight tracks projecting them into silicon planes. The CVD diamond strip detectors of the telescope allowed us to perform particle tracking and study detectors under test. In a "transparent" analysis we measured the signal distribution in the silicon detectors and their residual distributions.

\section{REFERENCES}

[1] F. Borchelt et al. (RD42-Collaboration). "First Measurements with a Diamond Microstrip Detector". Nucl. Instr. Meth., A354 (1995).

[2] W. Adam et al. (RD42 Collaboration). "First Bump-Bonded Pixel Detectors on CVD Diamond". Nucl. Instr. Meth., A436 (1999) 326-335.

[3] W. Adam et al. (RD42 Collaboration). "Micro-Strip Sensors based on CVD Diamond". Nucl. Instr. Meth., $\mathbf{4 5 3}$ (2000) 141-148, or CERN-EP-2000041.

[4] W. Adam et al. (RD42-Collaboration). "Development of Diamond Tracking Detectors for High Luminosity Experiments at the LHC". Status Report/RD42, CERN/LHCC 2001-002, (2001).

[5] De Beers Industrial Diamond Division. Charters, Sunninghill, Ascot, Berkshire, SL5 9PX England.

[6] Integrated Detector and Electronics (IDEAS). "The VA Circuits". www . ideas. no. Pb.315, Veritasveien 9, N-1322 Hovik, Norway.

[7] W. Adam et al. (RD42 Collaboration). "Performance of Irradiated CVD Diamond Micro-Strip Sensors". Nucl. Instr. Meth., (2001), or CERN-EP2000-115.

[8] C. Colledani et al. "A Submicron Precision Silicon Telescope for Beam Test Purposes”. Nucl. Instr. Meth., A372 (1997) 3.

[9] D. Meier. "CVD Diamond Sensors for Particle Detection and Tracking". $\mathrm{PhD}$ thesis, University of Heidelberg and CERN, atlasinfo.cern.ch/Atlas/ documentation/thesis/thesis.html, (1999). 